\title{
Study on Hyperspectral Models in estimating Chlorophyll Content of Corn Based on Hyperspectral measured data and HJ-HSI
}

\author{
SHAO Tian-tian ${ }^{\mathrm{a}, \mathrm{b}}$ \\ a Northeast Institute of Geography and Agroecology, \\ Changchun 130102, China; b University of Chinese \\ Academy of Science, Beijing 10049, China
}

\author{
SONG Kai-shan, ZHANG Bai \\ Northeast Institute of Geography and Agroecology, \\ Changchun 130102, China
}

\begin{abstract}
Chlorophyll is one of the most important pigments for plant photosynthesis as a proxy of health of vegetation and photosynthetic capacity. In this study, estimation of chlorophyll content of corn of western regions of Jilin Province is conducted based on both the measured hyperspectral data and the environmental satellite hyperspectral image data (HJ-HSI). Four kinds of vegetation indices including NDVI, MSR, MCAVI/OSAVI and TCAVI/OSAVI, are used for the inversion of chlorophyll. Among these vegetation indices, NDVI performs the best in estimating chlorophyll, followed by MSR and TCAVI/OSAVI. Otherwise, using HSI data based on vegetation indices in modeling performs not very well but the verification accuracy is rather high $\left(R^{2}>0.9\right)$. And the slopes of validated models are all less than 0.4 , which indicate that these estimates are lower than the measured results.
\end{abstract}

Key words-Vegetation indices; HJ-HSI; three-band model; chlorophyll content

\section{INTRODUCTION}

Leaf chlorophyll content $(\mathrm{Chl})$, a good indicator of photosynthesis activity and nutritional state, is significant to precision agriculture. Chlorophyll is one of the most important pigments for plant photosynthesis as a proxy of health of vegetation and photosynthetic capacity. As a result, the exact inversion of the chlorophyll is an important symbol for crops growth stage and status as well.

Remote sensing technology has played a unique role in quantitative estimation and simulation of carbon and nitrogen in the crop ecosystem element cycling, diagnostic and regulating crop fertilizer. Hyperspectral remote sensing data owns more advanced features of spectral banddimensional narrow (1 10nm) and continuous spectral configuration that multi-spectral remote sensing cannot provide.

The objective of this study is to compare the performance of a set of hyperspectral vegetation indices in chlorophyll content estimation based on different bands combination of the reflectance. Two new vegetation indices are derived which have better linearity with chlorophyll content. In order to test the reliability of selected indices, Hyperspectral Imaging Radiometer (HSI) data is used in application of remote sensing to estimate the chlorophylls of corns in central and western regions of Jilin Province.

\section{MATERIALS AND METHODS}

\section{A. Study Area Description and Data Acquisition}

The reflectance spectra and corn chlorophyll determination samples were collected in central and western regions of Jilin Province. The study area is the main region of corn production of China.

Reflectance spectra were obtained with a FieldSpec FR spectroradiometer (ASD, Inc., Boulder, CO) measuring reflectance over a spectral range of 350-2500 nm, and the spectral interval is $1 \mathrm{~nm}$. The concentration per-unit mass of $\mathrm{Chl}(\mathrm{mg} / \mathrm{g})$ were measured by spectrophotomete using acetone $(80 \% \mathrm{v} / \mathrm{v})$ as a solvent and employing the equations of Lichtenthaler (Lichtenthaler et al., 1996). Totally, 142 groups of in situ measured data are collected in the study area.

HSI is the first civilian spaceborne hyperspectral image in China and acquires data in 115 bands covering the visible, near, and shortwave-infrared ranges. Moreover, the spatial resolution is 100m (Zhao et al., 2010; Meng et al., 2010). In this paper, the HSI data was acquired at august of 2011 and chlorophyll content of corn is collected at the same month. Radiometric and geometric corrections were performed before analysis for HSI data. Because of the limitation of the number of HSI image and the corresponding ground data, there are only 21 groups of chlorophyll content covered with HSI, as fig. 1 shows. $2 / 3$ of the data is used to establish models and the others serve as validated data.

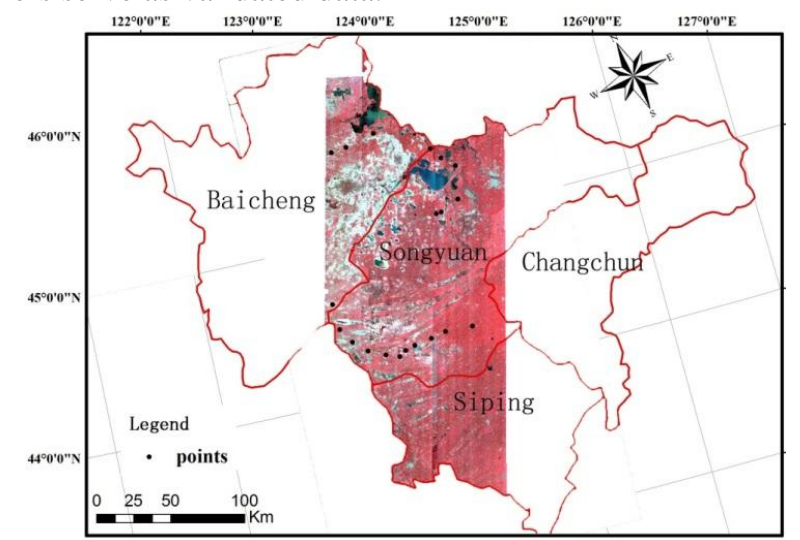

Fig. 1 Location of the points covered with HSI in the central and western regions of Jilin Province 


\section{B. Modeling Approaches}

Numerous investigations have conducted on estimating the physiological parameters based on the vegetation indices (Song et al, 2008; Gitelson et al., 2006; Dong et al., 2009). Spectral vegetation indices use the characteristic shape of the green vegetation spectrum by combining the low reflectance in the

\section{RESULTS AND DISCUSSION}

\section{A. Correlation between single band and chlorophyll content}

In order to make sure the sensitive between each band and chlorophyll content, analysis of reflectance of single band and first derivative of spectra are conducted before other methods used.

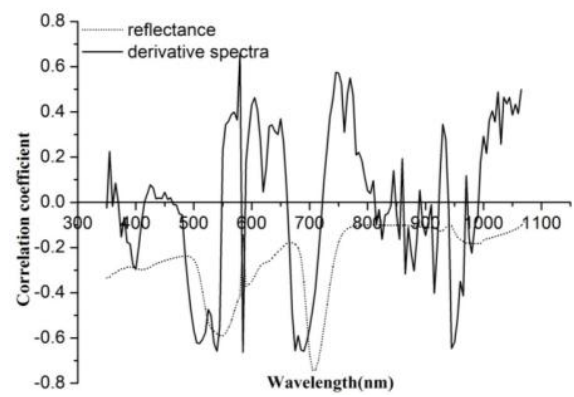

Fig. 2 Location of the points covered with HSI in the central and western regions of Jilin Province visible wavelength with the high reflectance in near-infrared wavelength (Wu et al., 2008). Six kinds of indices including NDVI, RVI, MSR, MCAVI/OSAVI and TCAVI/OSAVI are used in this paper (Rouse., 1974; Slater and Jacson, 1982; Rondeaux et al., 1996; Wu et al., 2008)

As fig. 2 shows, correlation coefficient of the spectral reflectance and chlorophyll is relatively stable in the entire wavelength range, showing a negative correlation. The maximum value is 0.741 at $710 \mathrm{~nm}$ belonging to red bands. The volatility of correlation coefficient, between first derivative of spectrum and chlorophyll content, is relatively larger in the entire wavelength range compared with that of reflectance and chlorophyll content. The maximum value is 0.658 at $540 \mathrm{~nm}$ belonging to green bands and the best correlation belongs to NIR is located in $750 \mathrm{~nm}$.

\section{B. Regression between chlorophyll and vegetation indices}

On the basis of vegetation indices talked above, we can get established models and $\mathrm{R}^{2}$ listed in Table 1 . Obviously, some vegetation indices (RVI and DVI, with the $\mathrm{R}^{2}$ lower than 0.3 ) are not suitable to estimate chlorophyll content with the ground data getting from Jilin Provence.

TABLE I. MODELS FOR ESTIMATING CHLOROFHYLL CONCENT BASED ON VARIOUS VEGETATION INDICES

\begin{tabular}{cccc}
\hline Vegetation indices & Wavebands $(\mathrm{nm})$ & Models & $\mathrm{R}^{2}$ \\
\hline NDVI & $670 、 800$ & $\mathrm{y}=4.148 \mathrm{x}^{3.419}$ & 0.330 \\
RVI & $670 、 800$ & $\mathrm{y}=0.5019 \mathrm{x}^{0.593}$ & 0.259 \\
MSR & $670 、 800$ & $\mathrm{y}=0.767 \mathrm{x}^{0.951}$ & 0.279 \\
DVI & $670 、 800$ & $\mathrm{y}=3.4088 \mathrm{x}^{0.341}$ & 0.037 \\
MCAVI/OSAVI & $540 、 710 、 740$ & $\mathrm{y}=2.295 \mathrm{x}^{0.904}$ & 0.346 \\
TCAVI/OSAVI & $540 、 710 、 740$ & $\mathrm{y}=-5.091 \mathrm{x}+4.870$ & 0.563 \\
\hline Optimized NDVI & $710 、 740$ & $\mathrm{y}=0.366 \mathrm{e}^{4.075 \mathrm{x}}$ & 0.717 \\
Optimized MSR & $710 、 740$ & $\mathrm{y}=2.844 \mathrm{x}^{1.193}$ & 0.716 \\
\hline
\end{tabular}

yield high accuracy compared to the original models. As a

It also shows that the models based on those original indices perform poorly for estimating chlorophyll content (TABLE I). On the contrary, the chlorophyll estimation based on the optimized models due to more sensitive bands
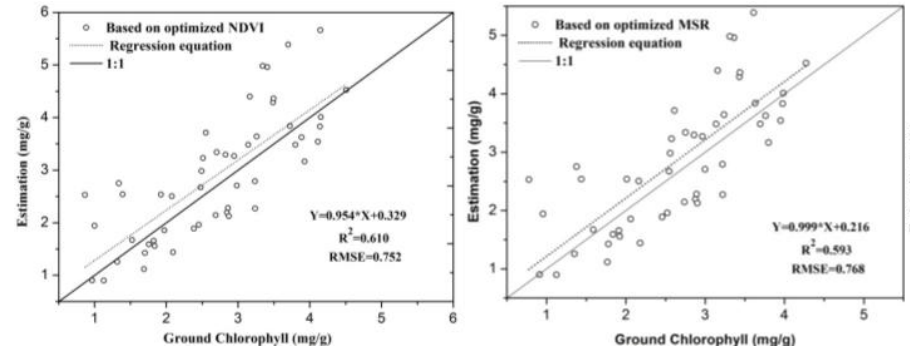

Fig. 3 Validation of models based on different vegetation indices

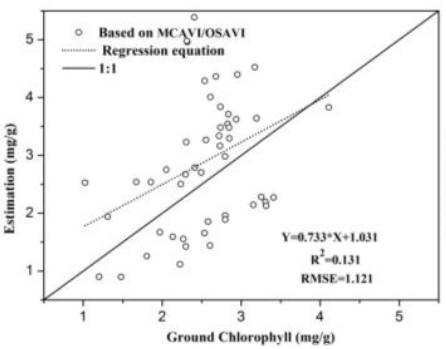
result, models based on optimized indices are used to estimate chlorophyll content and the RVI and DVI are eliminated. As fig.3 shows, 49 groups of data are selected to validate the models listed in TABLE I.

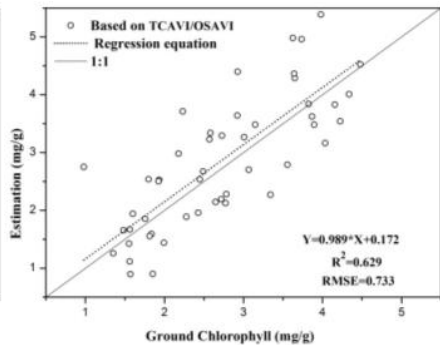

Among the four indices, MCAVI/OSAVI performs not very well which is not consistent with other studies where MCAVI/OSAVI is well used in estimating chlorophyll
(Rondeaux et al., 1996; Daughtry et al., 2000; Wu et al., 2008). Moreover, the result is in agreement with the study, which confirms that using TCAVI/OSAVI is better than 
MCAVI/OSAVI (Haboudane et al., 2002). Furthermore, NDVI optimized by the actual data is always stable no

\section{Validation of vegetation indices with HSI and ground data}

Among For the vegetation indices used in the chlorophyll inversion with the ground data above, the accuracies obtained from NDVI and MSR are better than others. However, the accuracy based on optimized vegetation indices is somehow improved compared with those use original spectral bands. Therefore, NDVI, MSR matter modeling or validating.

and their optimized forms are selected to estimate chlorophyll using HSI.

According to the sensibility analysis between single band and chlorophyll content, $710 \mathrm{~mm}$ and $740 \mathrm{~mm}$ are more suitable to establish models. The B079 of HSI image is corresponding to $\mathrm{R}_{710}$. Since no HSI band centered at 740 $\mathrm{nm}$, two relative bands (B084 and $\mathrm{B} 085$ ) are chosen to calculate $\mathrm{R}_{740}$ with the formula below:

$$
\mathrm{R}_{740}=0.5\left(\mathrm{R}_{\mathrm{B} 084}+\mathrm{R}_{\mathrm{B} 085}\right)
$$

TABLE II. THE COMPARISON BETWEEN ORIGINAL INDICES AND REVISED INDICES

\begin{tabular}{cccc}
\hline Vegetation indices & Bands selected & Prediction models & $\mathrm{R}^{2}$ \\
\hline Original NDVI & $670 \mathrm{~mm}, 800 \mathrm{~mm}$ & $\mathrm{y}=3.863 \mathrm{x}^{0.464}$ & 0.451 \\
Original MSR & $670 \mathrm{~mm}, 800 \mathrm{~mm}$ & $\mathrm{y}=0.703 \ln (\mathrm{x})+2.813$ & 0.329 \\
Revised NDVI & $710 \mathrm{~mm}, 740 \mathrm{~mm}$ & $\mathrm{y}=4.712 \mathrm{x}^{0.357}$ & 0.415 \\
Revised MSR & $710 \mathrm{~mm}, 740 \mathrm{~mm}$ & $\mathrm{y}=0.719 \ln (\mathrm{x})+3.577$ & 0.336 \\
\hline
\end{tabular}

As far as Table II concerned, the $\mathrm{R}^{2}$ of prediction models based on the original indices, range from 0.329 for MSR[670,800] to 0.451 for NDVI[670,800]. Although revised hyperspectral vegetation indices have higher correlation coefficients than its counterparts using measured
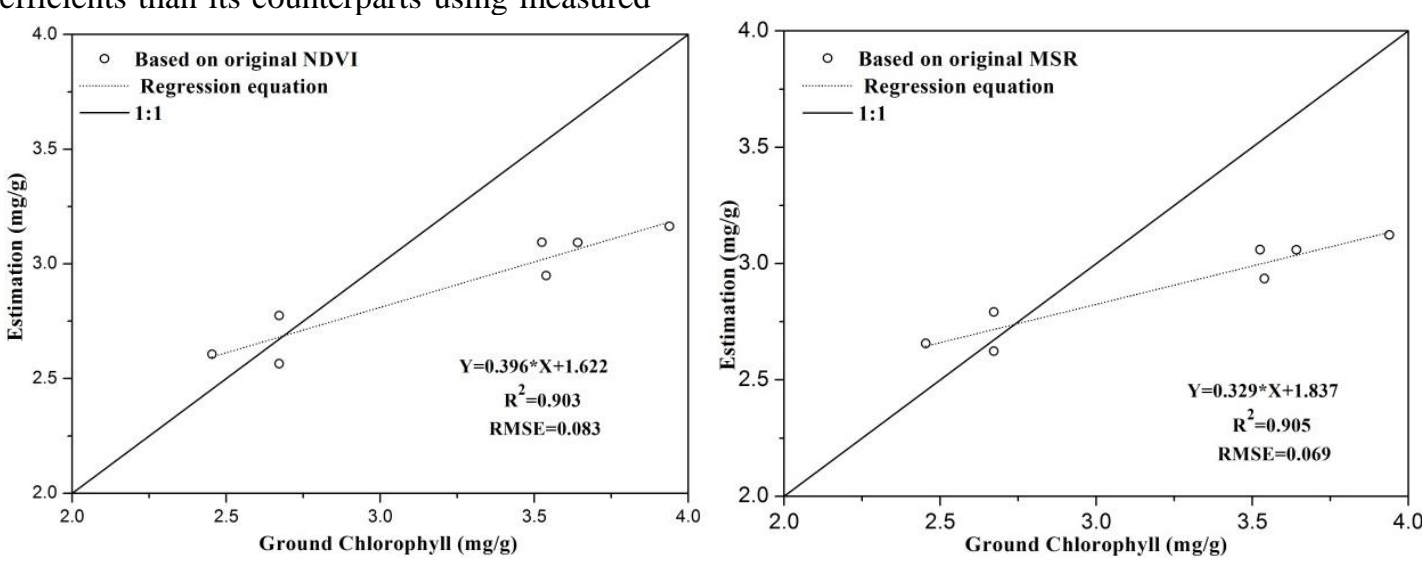

Fig.4 Performs of estimating models based on HSI image
As shown in Fig. $4, \mathrm{R}^{2}$ of validation models based on original MSR and NDVI are rather high, 0.905 and 0.903 respectively. But the slopes of validation models are less than 0.4, which indicates that the predictions of $\mathrm{Chl}$ are lower than the actual data. The main reasons for the high accuracy of validating models and low accuracy of estimating models is that the validated data is only 7 and the regressive models are built based on 14 groups of data. The representation of those data may be not enough to cover all affected by the underlying soil reflectance and the nonphotosynthetic materials (Wu et al, 2008), which lead to the low accuracy of regressive models and the high estimation models. the different types of the background. And the data is ground data, the new indices are not opting for chlorophyll content estimation based on HSI data. As a result, original indices (NDVI, MSR) are chose to validate the measured data and HSI data.

\section{CONCLUSIONS}

In this study, the analysis of chlorophyll content is conducted based on both the measured hyperspectral data of corn and the environmental satellite hyperspectral image data (HJ-HSI) based on high spectral vegetation indices to retrieve the leaf chlorophyll content of corn with measured data.

Four kinds of original vegetation indices, such as NDVI, MSR, MCAVI/OSAVI and TCAVI/OSAV do not perform well in estimating chlorophyll content. But the optimized NDVI and MSR gets better result than the original ones with in-situ measured data.

The results of using vegetation indices methods in the inversion of chlorophyll content based on HSI image are permissible. The coefficient of determination of the model in the modeling is not high, but the verification accuracy is 
rather high $\left(\mathrm{R}^{2}>0.9\right)$. And the slope of validated models is less than 0.4 , which indicates that estimates are lower than

\section{REFERENCES}

[1] E. Baret and G. Guyot, Potentials and limits of vegetation indices. Remote Sensing of Environment, 1991, 35, 161-173

[2] J.J. Dong, L. Wang and Z. Niu, Estimation of Canopy Chlorophyll Content Using Hyperspectral Data. Spectroscopy and Spectral Analysis, 2009, 29: 3003-3006 (In Chinese)

a) Gitelson, P. Keydan, M. Merzlyak, et al, Three-band model for noninvasive estimation of chlorophyll, carotenoids, and anthocyanin contents in higher plant leaves. Geophysical Research Letters, 2006, 33, L11402

[3] D. Haboudane, R. John, J. Millera, et al, Integrated narrowband vegetation indices for prediction of crop chlorophyll content for application to precision agriculture. Remote Sensing of Environment, 2002, 81, 416-426

[4] H.K. Lichtenthaler, A.A. Gitelson, and M. Lang. Nondestructive determination of chlorophyll content of leaves of a green and an aurea mutant of tobacco by reflectance Mmeasurements. Journal of Plant Physiology, 1996, 148:483493

[5] J.H. Meng, B.F. Wu, L.M. Niu, et al, Validation of HJ-1 HSI Red Edge Parameters Based on Spectral Reflectance of Hyperion. Spectroscopy and Spectral Analysis, 2010, 30: 2205-2210 (In Chinese)

[6] G. Rondeaux, M. Steven and F. Baret. Optimization of soil adjusted vegetation indices. Remote Sensing of Environment, 1996, 55: 95-107 the measured results.

[7] J.W. Rouse, R.H. Haas, J.A. Schell, et al, Monitoring the vernal advancements and retrogradation of natural vegetation. In: NASA/GSFC, Final Report, Greenbelt, MD, USA, 1974, $1-137$

[8] P.N. Slater and R.D. Jackson, Atmospheric effects on radiation reflected from soil and vegetation as measured by orbital sensors using various scanning directions. Applied Optics, 1982, 21: 3923-3931

[9] K.S. Song, B. Zhang, Z.M. Wang, et al, Estimation of chlorophyll-a concentration for soybean based on wavelet analysis. Journal of Plant Ecology, 2008, 32(1), 152-160 (in Chinese)

[10] C.Y. Wu, Z. Niu, Q. Tang, et al, Estimating chlorophyll content from hyperspectral vegetation indices: modeling and validation. Agricultural and Forest Meteorology, 2008, 148, $1230-1241$

[11] X. Zhao, J. Lu, A. Gong, et al, Operational spectrum reconstruction of data from the fourier transform hyperspectral imager onboard HJ-1A satellite. Chinese Science Bulletin, 2010, 55: 1808-1812

[12] C.S. Daughtry, C.L. Walthall and M.S. Kim, Estimating corn leaf chlorophyll concentration from leaf and canopy reflectance. Remote Sensing of Environment, 2000, 74(2): 229-239 